\title{
A Study on Implementation of Network Based Telemetry System Using Retransmission
}

\author{
Sooyeol Im, Chiho Hwang, Hwansuk Lee, Inho Choi \\ Agency for Defense Development, Korea, \\ syim@add.re.kr
}

\begin{abstract}
:
In recent years, a network based telemetry system has received a great attention as the upcoming telemetry paradigm. This paper considers a network based telemetry system with one master telemetry and two slave telemetries. The master telemetry merges data from each slave telemetry and own measured data, and the merged data is transmitted to the ground receiving station. If there exist some errors in data from the salve telemetry, the master telemetry requests retransmission to corresponding slave telemetry. In this paper, we propose a retransmission algorithm applied to implementing the network based telemetry system. Simulation results demonstrate that the proposed retransmission algorithm improves the performance of the wireless communication between the master and slave telemetries.
\end{abstract}

Key words: Telemetry System, Retransmission, Additive White Gaussian Noise, Bit Error Rate, Frame Error Rate

\section{Introduction}

The newly developed flight vehicles should confirm the stability and suitability of the design parameters by analyzing the flight test results. The telemetry system, in the flight test for the performance evaluation of the flight vehicle, acquires data from sensors attached to the flight vehicle and status of subsystems, and transmits the measured data to the ground receiving station via wireless communications [1]. The standardization of the telemetry system is actively in progress by Range Commanders Council (RCC) and Inter Range Instrumentation Group (IRIG) [2].

In recent years, a network based telemetry system has received a great attention as the upcoming telemetry paradigm due to the applicability to the group of flight vehicles [3], [4]. In the formation of group of flight vehicles, each flight vehicle has its own on-board telemetry, and the on-board telemetries and ground receiving station are networked via wireless communications. Integrated Network Enhanced Telemetry (iNET) project of Central Test and Evaluation Investment Program (CTEIP) is actively on defining the concept and requirements of the system, defining the network and communication protocol, incorporating standardized technologies [5], [6].
In the flight test of the group of flight vehicles, each telemetry has been allocated frequency band separately using frequency division multiplexing (FDM). In such a telemetry system, in accordance with the number of flight vehicles, there should be multiple frequency bands and multiple wireless receiving system in the ground receiving station.

This paper considers a network based telemetry system with multiple on-board telemetries. One on-board telemetry (master telemetry) receives data from multiple on-board telemetries (slave telemetries) acquiring its own measured data. Slave telemetries transmit the measured data to the master telemetry in turn using time division multiplexing (TDM) over one frequency band. The master telemetry merges the data from slave telemetries and its own data, and the merged data is transmitted to the ground receiving station. In such a network, the retransmission scheme is applied to the telemetry system via the bidirectional communication link between the master and slave telemetries, and we propose a retransmission algorithm determining which slave telemetry retransmits which error-frame. Simulation results demonstrate that the proposed retransmission algorithm improves the performance of bit error rate (BER) and frame error rate (FER) between the master and slave telemetries. 


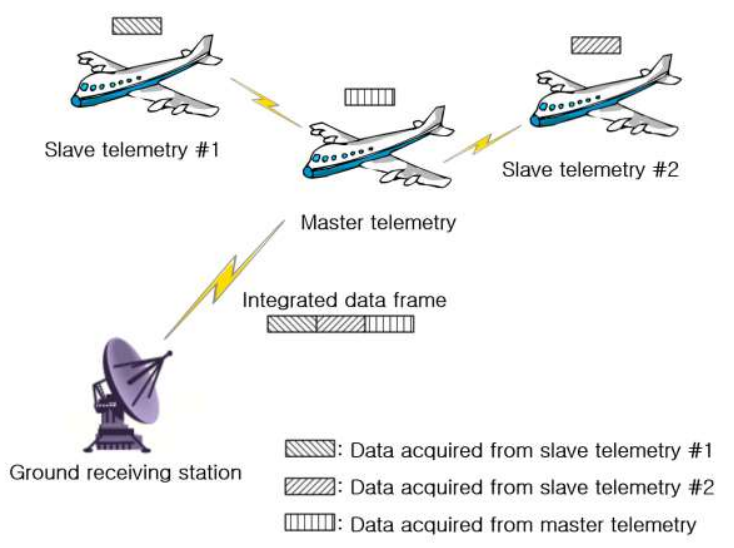

Fig. 1. Network based telemetry system.

The remainder of this paper is organized as follows. In Section II, we introduce the system model applied in this paper. Details of the proposed retransmission algorithm are given in Section III. In Section IV, some simulation results are provided to demonstrate the performance of the proposed retransmission algorithm. Finally, this paper is concluded in Section V.

\section{System Model}

This paper considers a network based telemetry system with one master telemetry and two slave telemetries as shown in Fig. 1. Each slave telemetry acquires and transmits the measured data to the master telemetry. The master telemetry merges and transmits the data from slave telemetries and its own data to the ground receiving station.

The master telemetry and two slave telemetries take a data transmission using TDM. One cycle of the data transmission is shown in Fig. 2. The master telemetry broadcasts the data request command in each cycle. Each salve telemetry, received the data request command from the master telemetry, transmits the acquired data to the master telemetry at a predefined time. In such a procedure, each slave telemetry transmits the data measured at the same time. In case that there are some errors in the data received by the master telemetry, the master telemetry inserts the retransmission request into the data request command. The slave telemetry, received the data request command containing the retransmission request, retransmits the corresponding data frame to the master telemetry. The number of retransmission within one cycle is defined in accordance with the circumstance of the telemetry system such as wireless communication bandwidth, the amount of data acquired by the slave telemetry.
Slave telemetry \#1 Master telemetry Slave telemetry \#2

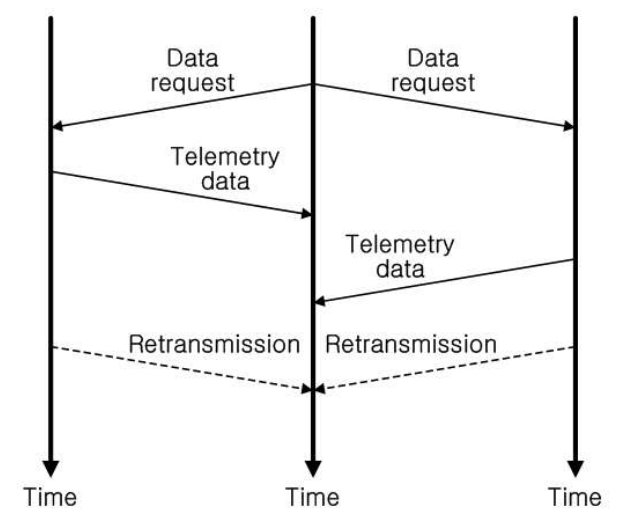

Fig. 2. Data transmission between the master telemetry and slave telemetries in the network based telemetry system.

\section{Retransmission Algorithm}

In this section, we explain the retransmission algorithm for data retransmission of the slave telemetry in the network based telemetry system. Since one of two slave telemetries retransmits the measured data in a cycle, it is necessary to determine which slave telemetry retransmits the data in corresponding cycle.

The master telemetry executes the retransmission algorithm and inserts the result of the retransmission algorithm into the data request command in corresponding cycle. For the retransmission algorithm execution, in this paper, it is assumed that the master telemetry has two status registers about the data received from each slave telemetry. The bits of the status register are shifted when receiving the data frame from the slave telemetry, and the corresponding bit of the corresponding status register is set in accordance of the result of error detection such Cyclic redundancy code $(\mathrm{CRC})$. If there exists error in the received data, the corresponding bit of the corresponding status register is set to 1 . Otherwise, the corresponding bit is set to 0 . The length of the status register is determined in accordance with the size of the memory of the slave telemetries due to the number of data frames stored in the memory of slave telemetries depends on the length of the status register.

The flowchart of the retransmission algorithm executed at the master telemetry is shown in Fig. 3. By choosing the slave telemetry with larger status register value, let the slave telemetry with older error-frame preferentially retransmits the corresponding frame. The retransmission algorithm proposed in this paper allows $\mathrm{N}$-times consecutive retransmission using RetriLock variable. In case that a slave 


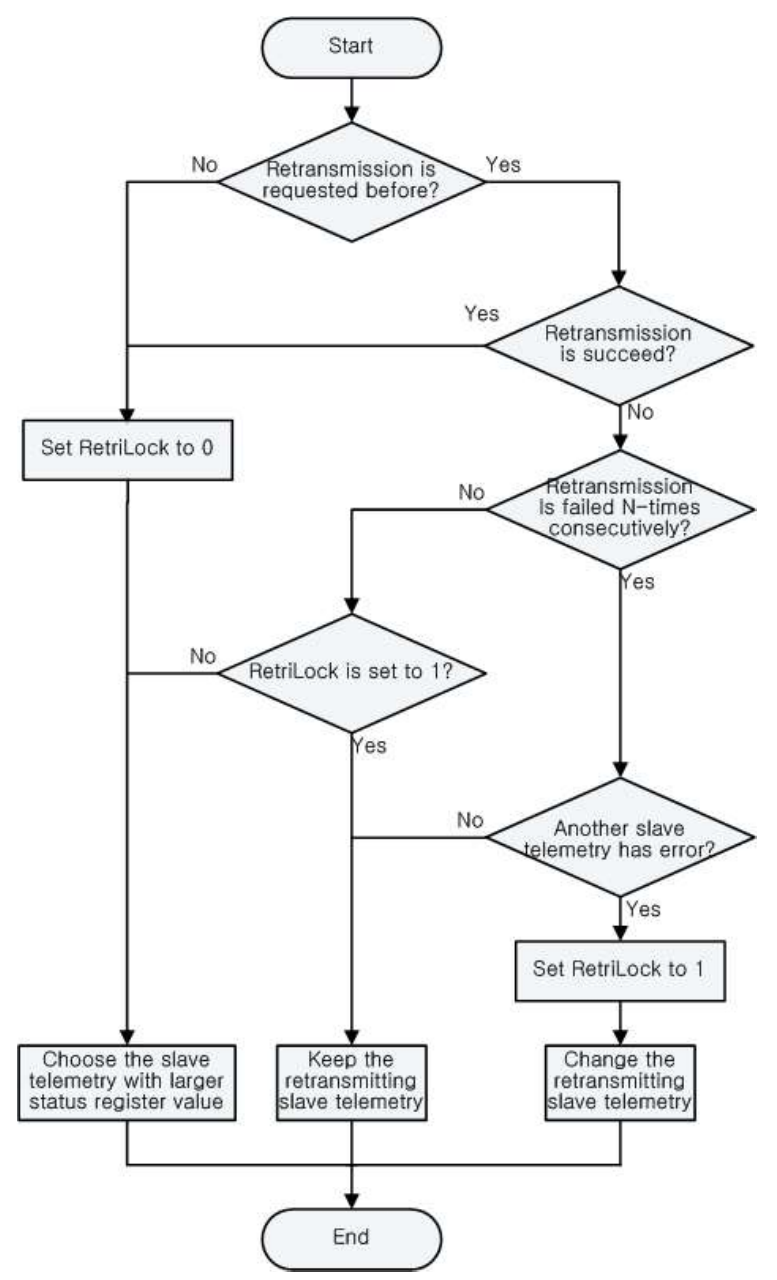

Fig. 3. Flowchart of retransmission algorithm.

telemetry fails retransmission $\mathrm{N}$-times consecutively, RetriLock is set to 1 , and the retransmitting slave telemetry is changed in the next cycle. As a result of the retransmission algorithm, the master telemetry inserts the information about which slave telemetry retransmits which data frame, using the status register value, into the data request command.

\section{Performance Evaluation}

In this section, some simulation results are provided to demonstrate the proposed retransmission algorithm. We consider the network based telemetry system that two slave telemetries transmit each acquired data and one master telemetry receives the data. Each slave telemetry modulates the acquired data using frequency shift keying (FSK). For the sake of simplicity, it is assumed that the wireless channel between the master and slave telemetries is modeled as Additive White Gaussian Noise (AWGN) channel, and the data frame is retransmitted once a cycle. It is further assumed that the length of the status register is

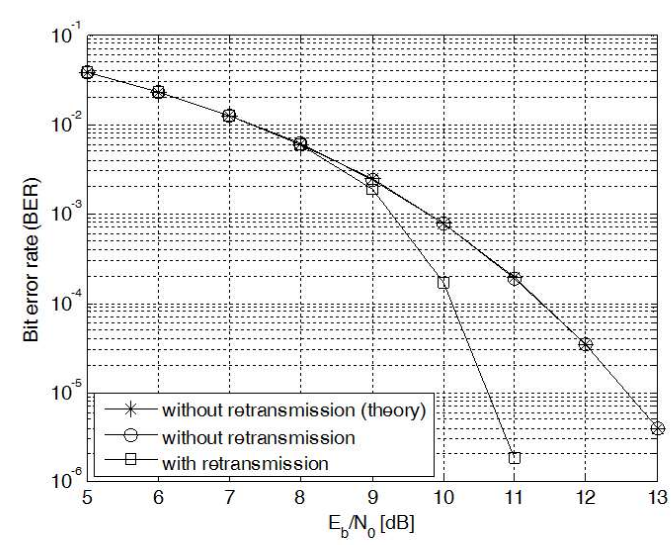

Fig. 4. Comparison of BER performance of FSK modulation with/without retransmission over AWGN channel.

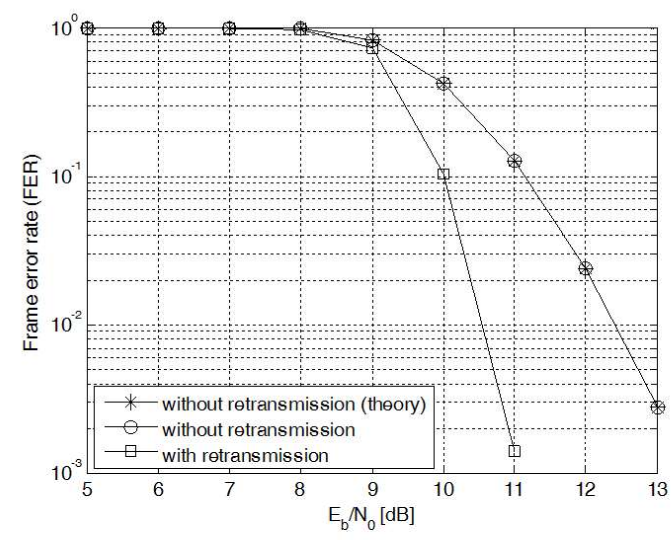

Fig. 5. Comparison of FER performance of FSK modulation with/without retransmission over AWGN channel.

16 bits, and the length of the data frame is 88 bytes.

We change the retransmitting slave telemetry in case that the corresponding slave telemetry fails the retransmission twice consecutively.

Fig. 4 presents the BER performance of FSK modulation over AWGN channel. For performance comparison, the BER performance of the FSK without retransmission over AWGN is plotted. The result can be represented using Q-function as follows [7]:

$$
\begin{aligned}
P_{b} & =Q\left(\sqrt{\frac{E_{b}}{N_{0}}}\right) \\
& =\frac{1}{\sqrt{2 \pi}} \int_{\sqrt{\frac{E_{b}}{N_{0}}}}^{\infty} \exp \left(-\frac{x^{2}}{2}\right) d x .
\end{aligned}
$$

In the range of low $E_{b} / N_{0}$, as shown if Fig. 4, the retransmission algorithm does not influence 
the BER performance. In the simulation environment of this paper, the retransmission algorithm enhances the BER performance in the range of $E_{b} / N_{0} \geq 8 \mathrm{~dB}$, and the influence of the retransmission algorithm gets higher with increasing $E_{b} / N_{0}$. In general, reliable communication can be achieved at $10^{-5}$ of BER [8], [9], and the proposed retransmission algorithm obtains $2 \mathrm{~dB}$ of $E_{b} / N_{0}$ gain at $10^{-5}$ of BER compared to the conventional scheme without retransmission.

Fig. 5 presents the FER performance of FSK modulation over AWGN channel. For performance comparison, the FER performance of the FSK without retransmission over AWGN is plotted. Since we assumed that the length of the data frame is 88 bytes (i.e., 704 bits), theoretical FER can be represented by the Qfunction, using eq. (1), as follows:

$$
\begin{aligned}
P_{f} & =1-\left(1-P_{b}\right)^{704} \\
& =1-\left(1-Q\left(\sqrt{\frac{E_{b}}{N_{0}}}\right)\right)^{704} \\
& =1-\left(1-\frac{1}{\sqrt{2 \pi}} \int_{\sqrt{\frac{E_{b}}{N_{0}}}}^{\infty} \exp \left(-\frac{x^{2}}{2}\right) d x\right)^{704} .
\end{aligned}
$$

In the range of low $E_{b} / N_{0}$, like the result of BER in Fig. 4, the retransmission algorithm does not influence the FER performance. With increasing $E_{b} / N_{0}$, however, the influence of the retransmission algorithm gets higher. In the range of $E_{b} / N_{0} \geq 9.5 \mathrm{~dB}$ (i.e., BER is less than $1 / 704 \approx 1.42 \times 10^{-3}$ ), deterministically, there exist data frames without error due to none of bits within one frame has error. In the statistical simulation results of this paper, in the range of $E_{b} / N_{0} \geq 8 \mathrm{~dB}$ (i.e., BER is less than $6 \times 10^{-3}$ ), there exist data frames without error, and the influence of the retransmission algorithms arises.

\section{Conclusion}

This paper studied the implementation of the network based telemetry system with one master telemetry and two slave telemetries. In such a scenario, the retransmission algorithm is applied to the telemetry system to enable the retransmission of the error-frame via the bidirectional communication link. To this end, we proposed the retransmission algorithm determining which slave telemetry retransmits to the master telemetry. Simulation results demonstrated that the proposed retransmission algorithm enhances the performance of the BER and FER over AWGN channel compared to the results without retransmission.

By extending our work, the proposed retransmission algorithm can be generalized for the case that there exist multiple slave telemetries (i.e., not two slave telemetries, but $\mathrm{N}$-slave telemetries), and more realistic wireless channel model (e.g., Rayleigh, Rician channel model) can be applied in evaluating and analyzing the performance of the proposed retransmission algorithm. Furthermore, mathematical derivation of the BER and FER can be obtained in a given channel model, and the theoretical results can be compared with the statistical simulation results.

\section{References}

[1] F. Garden, R. Jedlicka, and R. Henry, Telemetry Systems Engineering, Artech House, 2002.

[2] RCC, Telemetry Standards, IRIG Standard, 10615, July 2015.

[3] S. Kim, S. Cho, S. Cho, and C. Park, "Development of operation network system and processor in the loop simulation for swarm of small UAVs," J. Inst. Contr. Robot. Syst., vol. 18, no. 5, pp. 433-438, May 2012.

[4] S. Kim and Y. Kim, "Behavioral decentralized optimum controller design for UAV formation flight," J. of The Korean Society for Aeronautical \& Space Sciences, vol. 36, no. 6, pp. 565-573, June 2008.

[5] T. Grace, J. Kenney, M. Moodie, and B. Abbot, "Key components of the iNET test article standard," in Proc. Int. Telemetering Conf., pp. 110, 2009.

[6] B. Abbot, M. Moodie, T. Newton, and T. Grace, "iNET system design concepts," in Proc. Int. Telemetering Conf., pp. 1-9, 2011.

[7] S. Haykin, Digital Communications, John Wiley \& Sons, 1988.

[8] D. Costello, J. Hagenauer, H. Imai, and S. Wicker, "Applications of error-control coding," IEEE Trans. Inform. Theory, vol. 44, no. 6, pp. 2531-2560, Oct. 2988.

[9] J. Proakis, Digital Communications, McGraw-Hill, 2000. 